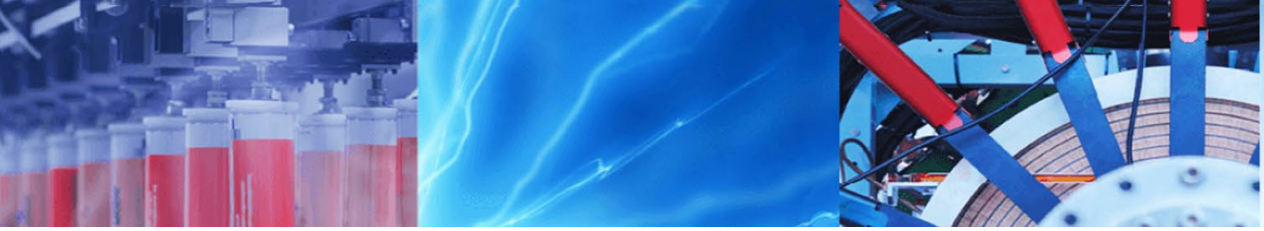

Research Article

\title{
Investigating the residual characteristics of dryer lint for developing resource recovery strategies
}

\author{
Pravin Kannan ${ }^{1}$ (D) Fawzi Banat ${ }^{1}$
}

Received: 13 August 2020 / Accepted: 5 October 2020 / Published online: 30 October 2020

(c) Springer Nature Switzerland AG 2020

\begin{abstract}
The residual structural and morphological characteristics of the exfoliated textile fibers, termed as dry lint, trapped in commercial laundry dryer filters have been assessed for the first time. Any attempt toward resource recovery from lint would require detailed material characterization and properties as a priori. Lint fibers collected from five different commercial laundromat dryer filters were used as characteristic samples for the preliminary investigation. The residual morphological and molecular structural characteristics of lint fibers were evaluated using microscopic imaging, spectroscopy and diffraction methods. Microscopic imaging revealed the extent of fiber fibrillation and agglomeration as a result of textile laundering process. Fibrillation intensity could be realized in significant reduction of water retention value of bulk fibers that was measured as $0.45 \mathrm{~g} / \mathrm{g}$ of lint. Interestingly, characterization data from Fourier transform infrared spectroscopy (FT-IR) and X-ray diffraction (XRD) studies indicate that the crystalline and molecular structure of fibers were relatively less affected. Further, FTIR spectra and XRD diffractogram of lint fibers resemble that of virgin cellulose I polymorph, thereby signifying the presence of major proportions of cellulosic fibers in dry lint. Residual properties encourages the devise of efficient resource recovery strategies for engineering high-value-added products from lint waste, and thus curtail microfiber pollution.
\end{abstract}

Keywords Dryer lint $\cdot$ Textile fibers $\cdot$ Cellulosic fibers $\cdot$ Microstructural characteristics $\cdot$ Secondary recycling

\section{Introduction}

The degradation of fabric fibers is an economic challenge for laundering companies and contributes significantly to the microfiber pollution of marine and terrestrial bodies by the discharge of wastewater and solid waste disposal. One study reported that 35 percent of microplastic contaminants found in marine water environments belong to microsized fibers released from textile laundering effluent [2]. It is well known that due to the repeated wet laundering process, fabrics significantly lose fiber tensile strength and ultimately lead to fuzz and pill formation
[21]. The damaged fibers are loosened and released into the laundry wastewater effluent upon further laundering. Such wet fibers (also known as' wet lint') lead significantly to marine body microfiber emissions [5, 6, 23]. Moreover, during the drying process, few fibers are exfoliated from the fabric surfaces that get stuck in the filtration system of the dryer. These dry fibers, also referred to as dry lint, are not regarded as a valuable resource and are mostly disposed of in municipal solid waste streams. However, no accurate statistics on the volume and composition of lint produced or released from domestic or commercial laundromats are available. Only wet lint discharged into

Electronic supplementary material The online version of this article (https://doi.org/10.1007/s42452-020-03628-8) contains supplementary material, which is available to authorized users.

Pravin Kannan, pravin.kannan@ku.ac.ae; $₫$ Fawzi Banat, fawzi.banat@ku.ac.ae| 1 Department of Chemical Engineering, Khalifa University (SAN Campus), P.O. Box 127788, Abu Dhabi, United Arab Emirates. 
the wastewater effluent stream $[11,22,24]$ is considered by most environmental policies focused on implementing aquatic body pollution mitigation strategies. The trapped lint from dryers, however, has never been taken into account and viewed as a possible pollutant. A quick review of municipal lint handling systems shows that only a few established cities have adopted source segregation and collection-based waste management systems; see Table S1 in the supplementary section. The final fate of the lint fibers remains uncertain, despite a well-defined collection system, as they are disposed of in available postconsumer waste containers. Another most common, yet controversial, disposal practice involves blending with organic waste to prepare compost for soil enrichment. Owing to the presence of synthetic fibers in lint, the approach has largely been discouraged by environmental scientists. Dryer lint has been commonly used as a fire tinder as it consists of compacted fibers that are flammable. However, attempts at large-scale waste-to-energy recycling of lint have rarely been recorded due to synthetic fibers' prevalence. The development of low-cost adsorbents for wastewater treatment, packaging and construction materials, and compost or soil conditioners may be the subject of future resource recovery strategies.

The word "microfiber" has been defined in the textile industry as fine synthetic fibers having a diameter of less than $10 \mu \mathrm{m}[3,6,9]$. In the current study, for the sake of clarity, the word "dry lint" often referred to as "lint" denotes all dry fabric fibers trapped and retrieved from the filtration device of the dryer. During the washing process, cellulose-based fabrics are more vulnerable to deterioration than synthetic fibers. In comparison to $1 \mathrm{mg}$ of polyester fibers per gram of fabric during an accelerated wet laundering process, a recent quantification study estimated that cellulose-based fabrics shed as much as $4 \mathrm{mg}$ of microfibers [23]. To the authors' knowledge, there are no studies published in the literature that details the characterization of the dryer lint fibers. While the composition of lint might be inferred as a primary mixture of cellulose and synthetic fibers, the structure, morphology, and composition of (micro) fibers must be routinely studied.

The overall goal of this study is to gather knowledge that would be useful to enhance resource recovery strategies for dryer lint. The aims are to examine and compare the macroscopic and microscopic physicochemical properties of the discarded lint with virgin natural and synthetic fibers. Tests on water absorbability, morphology, and molecular structure would provide detailed material characterization on lint. The characterization dataset could be used to design fiber regeneration techniques for recycled fabrics, enhance laundry effluent treatment methodologies, and develop recycled fabrics for fiber regeneration. Furthermore, information about the spectrum of fibrous microplastics produced during the laundry drying process may be used in future studies to establish effective lint recycling and treatment strategies.

\section{Methods and materials}

\subsection{Material collection and processing}

Since the study requires significant volumes of lint fibers, household laundry dryers are not suitable as sample collection points. For this investigation, lint samples were collected from five different commercial laundromats in the city of Abu Dhabi, UAE. Roughly, about $50 \mathrm{~g}$ were collected from each unit, and later pooled together as sample aggregate. In each case, sample collection was achieved using the inbuilt filters (mesh size of ca. $50 \mu \mathrm{m}$ ) attached to the commercial dryers. Following multiple loading cycles averaged over a period of 3 days, the samples were gently removed from the filters and wrapped in aluminum foils before processed in the lab. Hence, the collected samples are not part of any one batch, but represent a mean sample averaged over multiple cycles and fabric types. The collected samples were physically mixed thoroughly so as to reduce any heterogeneity arising from different filters and loads. For each characterization study, three runs were performed using representative samples of ca. $10 \mathrm{~g}$ that was physically cleaned to remove human hair, threads, and other macrosize metal and plastic contaminants.

\subsection{Material preparation and characterization}

Fiber degradation or fibrillation during laundering process could be directly linked to the loss in degree of polymerization of the fibers. In order to evaluate the extent of fiber damage during the laundering process, water absorbency of lint was used as an indicative property as the ability of fibers to absorb water are highly influenced by the laundering process. For analyzing the morphology of lint fibers, optical and scanning electron microscopic techniques were employed at various magnifications. The molecular structural characteristics of lint fibers were studied using spectroscopic and diffraction methods. As part of the residual analysis, characterization data collected in the current study were compared to virgin textile fibers taken from literature.

\subsubsection{Water retention value (WRV)}

Water retention value (WRV) is a measure of the ability of fibers to retain water in the pore structure. WRV of lint was determined using DIN 53814 standard method wherein ca. $5 \mathrm{~g}$ of cleaned lint was soaked in about $250 \mathrm{ml}$ 
deionized water for $2 \mathrm{~h}$, centrifuged at $3000 \mathrm{rpm}$ or $20 \mathrm{~min}$ and oven dried at $105^{\circ} \mathrm{C}$ for $4 \mathrm{~h}$. The mass of centrifuged sample $\left(M_{w}\right)$ and oven dried sample $\left(M_{d}\right)$ provided an indication of the WRV of the lint sample which was determined as follows:

$\operatorname{WRV}(\%)=\frac{M_{w}-M_{d}}{M_{d}} \times 100$

\subsubsection{Microscopy studies}

A detailed physical and morphological characterizations of lint fibers would reveal constitutional fiber morphology, size distribution, and microscale fiber structure. Prior knowledge of the structure, properties, and volume of lint fibers could be used to design suitable filters to extract fibers from laundry effluent. Fiber size distribution was accomplished using Fluid Imaging Technologies Inc. FlowCam 8100. The imaging particle analysis and pattern recognition feature of FlowCam provides an automated method for characterizing the types and quantities of particles present. A dilute liquid sample (filtered through different sieves) containing lint fibers was introduced into the top of the flow cell through a cuvette using a microsyringe pump. Imaging and flow settings were adjusted to achieve optimal particle analysis. A high-speed digital camera photographed each particle as it flowed down the cell, recording particle count and concentration in real-time. Also, morphologies of lint sample were recorded at different magnifications using Hirox KH7700 digital light microscope and FEG QUANTA 250 scanning electron microscope (SEM) operating with an acceleration voltage of $5 \mathrm{kV}$.

\subsubsection{Spectroscopic analysis}

Transmittance spectra were assimilated using a Bruker Vertex 80v FTIR equipped with an attenuated total reflectance (ATR) accessory. Scans were recorded over the wave number range from 4000 to $600 \mathrm{~cm}^{-1}$ at an interaction angle of $45^{\circ}$ and a rapid scan rate of 110 spectra/s at $16 \mathrm{~cm}^{-1}$ spectral resolution. The spectra and the peaks were compared with Infrared and Raman Users Group (IRUG) spectral database index for pure cellulose and polyester fibers.

\subsubsection{Diffraction analysis}

X-ray diffraction (XRD) patterns of dryer lint were obtained using Panalytical X'Pert Pro XRD operated at a wavelength $1.54 \AA$ and $40 \mathrm{kV} / 40 \mathrm{~mA}$. The samples were scanned in fixed time (FT) mode with a counting time of $2 \mathrm{~s}$ under diffraction angle $2 \theta$ in the range of $1^{\circ}-60^{\circ}$. From the diffractogram, crystallite size and percent crystallinity were calculated. The crystallite size was calculated using the Scherrer equation: [12]

$D=K \lambda /(\beta \cos (\theta))$

where $D$ is the crystallites size in $\mathrm{nm}, K$ is the Scherrer constant (0.94), $\lambda$ is the wavelength of the $X$-ray source $(0.15406 \mathrm{~nm}), \beta$ is FWHM in radians, and $\theta$ is the peak position in radians. The $\%$ crystallinity (PC) was estimated using the following expression: [4]

$\mathrm{PC}=\left(\left(I_{002}-I_{a m}\right) / I_{002}\right) \times 100$

where $I_{002}$ and $I_{a m}$ refers to the peak intensities at a diffraction angle of $22.2^{\circ}$ and $18^{\circ}$, respectively.

\section{Results and discussion}

The process conditions encountered during fabric laundering significantly affect the fabric, and the exfoliated fibers could be expected to be deprived of the inherent fiber characteristics. Analyzing the structure and morphology of the lint fibers on different scales would assist in assessing the loss in physico-mechanical properties. Further, results from lint characterization could be used to better understand the effects of laundering on fabric fibers. Since lint fibers are subjected to varying intensities of repeated horrification in the laundering process, huge variations in fiber characteristics could be expected on a microscopic scale. These variations would be averaged in bulk scale characterization techniques and thus could be used as a reliable technique for property estimation. The current study is a preliminary investigation that analyzes fiber characteristics qualitatively.

\subsection{Water retention value (WRV)}

WRV is a measure of the swelling of cotton fibers when contacted with water. Since dryer lint comprises of a blend of various textile fibers including cotton cellulosic, WRV of the lint could be severely influenced by the repeated laundering process. Results from water retention value (WRV) tests of lint fibers are based on an average of five replicate runs employing different samples. Using Eq. (1), WRV of lint was estimated as $0.37 \mathrm{~g}$ of water per gram of lint with a standard deviation of \pm 0.01 between samples. The measurement error based on three tests using the same sample was \pm 0.005 , and hence, the analysis method was within acceptable limits. In comparison, the WRV of an unopened cotton ball was reported as $1.4 \mathrm{~g} / \mathrm{g}$ and that of field dried cotton was $0.46 \mathrm{~g} / \mathrm{g}$ [16]. As it can be seen, the water retention capacity by the fibers used in textiles 
undergone numerous laundering cycles is significantly less than once-dried cotton fibers. This could possibly be due to the chemical cross-linking in the fiber cell walls and internal pores which prevents them from swelling with water [8]. The low water holding capacity thus possibly indicates extensive fibril aggregation due to the irreversible collapse of pores in the fiber wall.

\subsection{Microscopic analysis}

The measured data on particle size and concentration using flow microscopy are provided in Table 1. It is interesting to note that although nearly $60 \%$ of the lint constitutional fibers are smaller than $10 \mu \mathrm{m}$ diameter, they contribute to only ca. $0.2 \%$ of the total sample volume. Roughly, $90 \%$ of the lint volume is composed of individual fibers larger than $50 \mu \mathrm{m}$ diameter. Figure 1 shows the aspect ratio scattergram that is plotted between aspect ratio (length/width) and equivalent spherical diameter (ESD). The measured aspect ratio of lint fibers is very small and could not be considered as a fiber resource for textile reprocessing applications which prefers a relatively higher aspect ratio on the order of hundreds [10]. A collage of representative images of various fibers present in lint has been shown in supplementary file, as Fig. S1. It can be noticed that the fibers are not of uniform shape and are extremely short to be termed as fibers. Moreover, analysis of lint revealed a diverse variety of fiber shapes, thereby revealing the heterogeneity within the lint.

The morphology of lint fibers was analyzed using microscopic photographs captured from optical (see Fig. 2) and scanning electron microscopes (see Fig. 3). While Fig. 2a illustrates a photograph of lint mass as-collected from commercial laundromat dryer filter, Fig. $2 b-d$ are pictures of lint at various magnifications captured using an optical digital microscope. It is evident that although

Table 1 Measured particle characterization data by flow microscopy

\begin{tabular}{lrlll}
\hline Filter $(\mu)$ & Count & Count $\%$ & Volume $\%$ & $\mathrm{P} / \mathrm{ml}^{\mathrm{a}}$ \\
\hline Total & 20,687 & 100 & 100 & 81,584 \\
$<10$ & 12,486 & 60.356 & 0.204 & 49,242 \\
$>10$ & 8201 & 39.643 & 99.796 & 32,343 \\
$>25$ & 3364 & 16.261 & 98.23 & 13,267 \\
$>50$ & 1210 & 5.849 & 91.45 & 4772 \\
$>75$ & 512 & 2.475 & 80.797 & 2019 \\
$>100$ & 233 & 1.126 & 68.987 & 919 \\
$>200$ & 28 & 0.135 & 35.913 & 110 \\
$>300$ & 7 & 0.034 & 18.458 & 28 \\
$>400$ & 1 & 0.005 & 5.035 & 3.94 \\
\hline
\end{tabular}

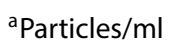

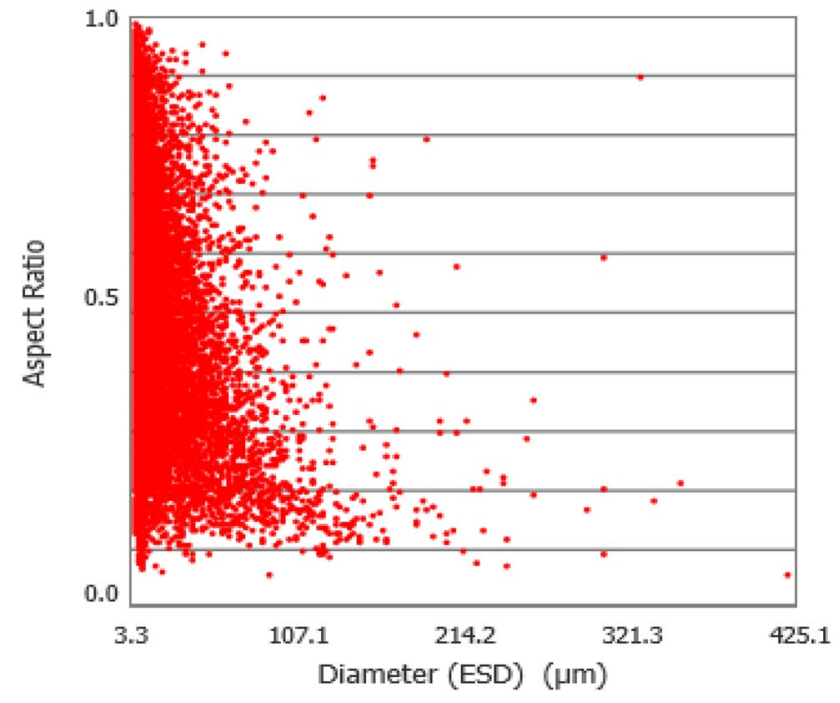

Fig. 1 Aspect ratio scattergram of lint fibers plotted against equivalent spherical diameter

lint comprises of fibers from different colored clothing, it resembles a fluff of bluish-gray tint. However, optical microscopy reveals the true physical morphology as an entanglement of numerous short natural and synthetic fibers, see Fig. $2 b$ at 50x magnification. At higher magnifications, the characteristic morphologies of individual fibers could be noticed. Figure $2 \mathrm{c}$ shows a single synthetic fiber with a smooth and uniform structure and an average diameter of $16 \mu \mathrm{m}$. Conversely, the characteristic twists and ribbon-like flat structure of cellulose fibers [19] is evident in Fig. $2 \mathrm{~d}$ captured at 2500x. The scanning electron microscopical images of lint, illustrated in Fig. 3a-d, reveals the fiber features, including type and morphology. Cellulosic fibers tend to exhibit fibrillation as a primary process sequence in addition to swelling during fiber breaking process [15]. The presence of microfibrils on cellulosic fibers in Fig. $3 c$ illustrates the extent of longitudinal fibrillation that is due to the mechanical abrasion caused by fiber swelling at wet conditions [25]. Also, it can be noticed that the width of the shredded fibers is in the same range as the original fibers typically employed for fabric yarning, but very short to be processed as textile fibers.

\subsection{FTIR spectral analysis}

To understand the ATR-FTIR spectra of lint fibers, it is important to understand the different functional groups usually present in textile fibers. As the predominant content of textile fibers is cellulose and other polysaccharides, the spectra of lint fibers could be expected to be superficially. 

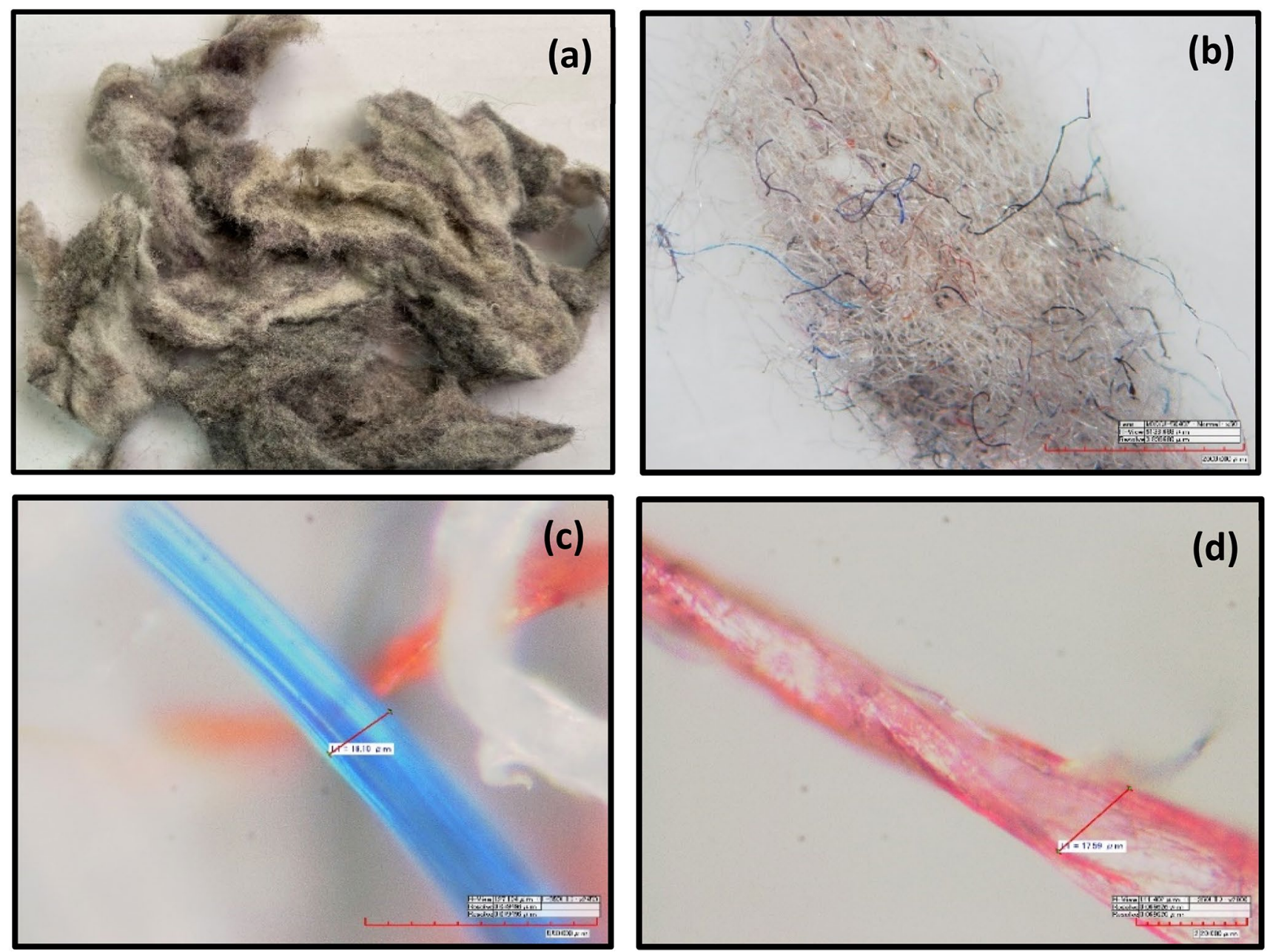

Fig. 2 a-d Light microscopy images of waste lint a lint collected from dryer, $\mathbf{b}$ magnified image at 50x, $\mathbf{c}$ a single dyed (blue) synthetic fiber at $2800 \times$, $\mathbf{d}$ a single dyed (red) cellulosic fiber at $2800 \times$

similar to natural cellulose. Figure 4 shows the spectra of lint fibers with a few dominant peaks that were compared with the spectrum of different textile fibers including wool, silk, polyamide, polyester, polyacrylic fiber, pure cellulose and viscose reported elsewhere [20]. As it can be easily confirmed, the ATR-FTIR spectra of lint show exception similarity based on characteristic peaks and band shapes to that of cellulose spectrum. For instance, the transmittance spectra of the lint exhibit a broadband in the region $3500-3200 \mathrm{~cm}^{-1}$ and peaking at $3323 \mathrm{~cm}^{-1}$ that could be interpreted as free $\mathrm{O}-\mathrm{H}$ stretching vibration of the hydroxyl groups in cellulosic fibers. In addition, the $\mathrm{C}-\mathrm{C}$ breathing band at $1160 \mathrm{~cm}^{-1}$ and $\mathrm{C}-\mathrm{O}-\mathrm{C}$ glycosidic ether band at $1104 \mathrm{~cm}^{-1}$ are signature peaks arising from polysaccharide components. Moreover, the $\mathrm{C}-\mathrm{H}$ symmetric stretching at $2897 \mathrm{~cm}^{-1}, \mathrm{O}-\mathrm{H}$ bending of the absorbed water at $1637 \mathrm{~cm}^{-1}, \mathrm{C}-\mathrm{H}$ bending at 1428 and $1363 \mathrm{~cm}^{-1}$, and $\mathrm{O}-\mathrm{H}$ in plane bending at $1314 \mathrm{~cm}^{-1}$ indicates that the cellulose fibers remains intact in the lint samples. Two additional peaks at 1711 and $718 \mathrm{~cm}^{-1}$ could be attributed to $\mathrm{C}=\mathrm{O}$ stretching and $\mathrm{C}-\mathrm{H}$ bending, respectively of polyester fiber present in the lint.

In order to specifically understand the effect of alkaline hydrolysis of laundering on textile fibers, selected intensity ratios of lint and cellulose fibers were compared in Fig. 5. The transmittance peak at $3,323 \mathrm{~cm}^{-1}$ corresponding to $\mathrm{O}-\mathrm{H}$ bond stretching was taken as the basis (base peak) and other intensity ratios were calculated as the ratio of peak intensity at a specific wave number to the peak intensity at the base peak. It can be noticed that most of the bands arising out of cellulose and polysaccharide components are impacted by the laundering process resulting in a reduction in molecular weight [13]. The impact is apparent from the intensity ratios observed at 1029 and $897 \mathrm{~cm}^{-1}$ corresponding to $\mathrm{C}-\mathrm{O}$ stretching in $\mathrm{C}-\mathrm{O}-\mathrm{H}$ alcohol fragments and glycosidic ether $(\mathrm{C}-\mathrm{O}-\mathrm{C})$ groups, respectively. The decrease is a direct consequence of the fibers' alkaline treatment during washing, causing 

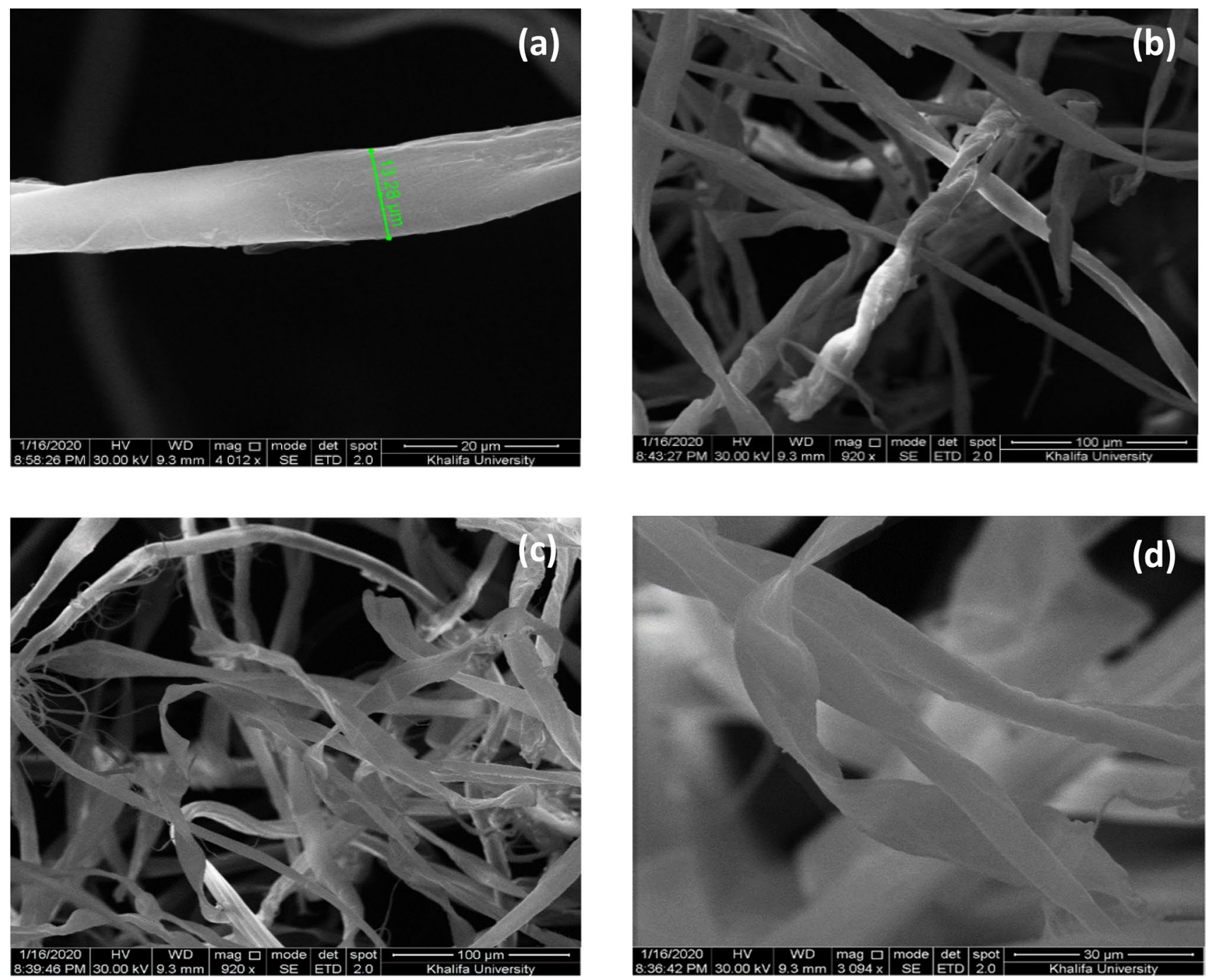

Fig. 3 a-d SEM images of waste lint a lint collected from dryer, $\mathbf{b}$ magnified image at $50 \times, \mathbf{c}$ a single dyed (blue) synthetic fiber at $2800 \times$, $\mathbf{d}$ a single dyed (red) cellulosic fiber at $2800 \times$

Fig. 4 ATR-FTIR spectra of lint sample from this study [orange lines indicates signature peaks of cellulose and green lines indicate polyester fibers] compared against IRUG spectral database

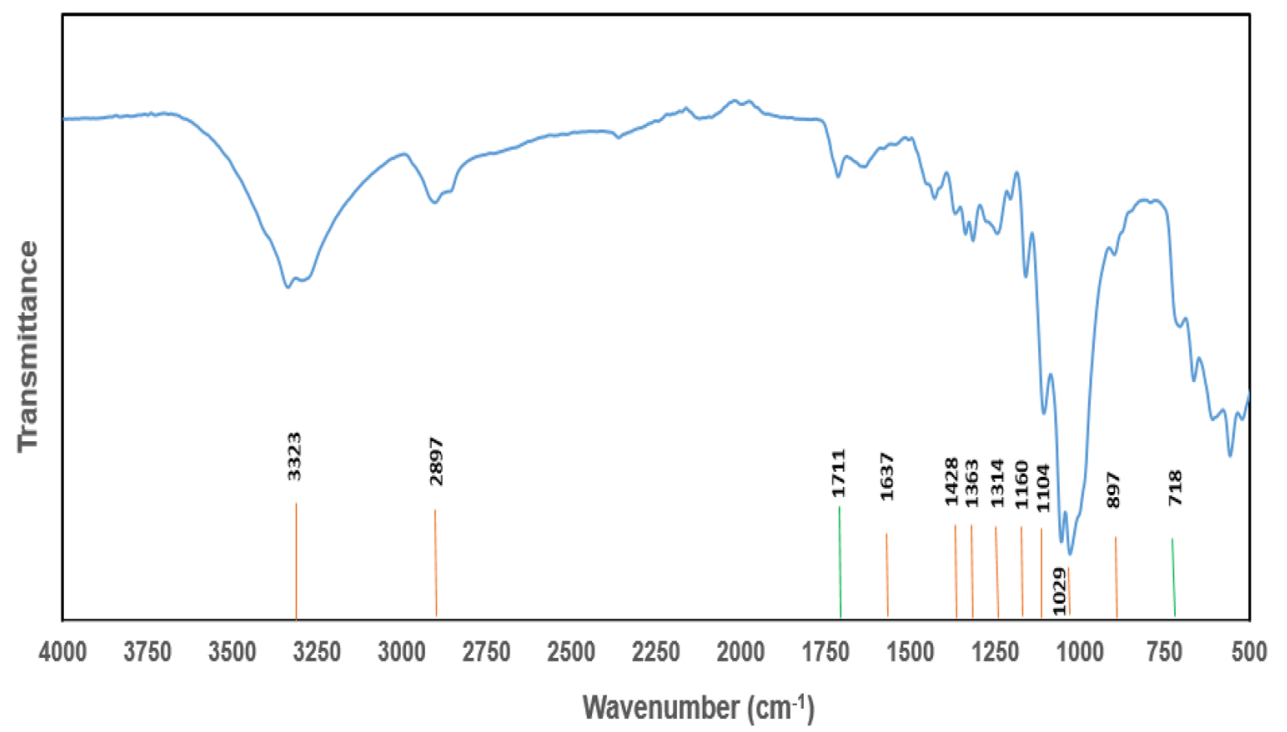




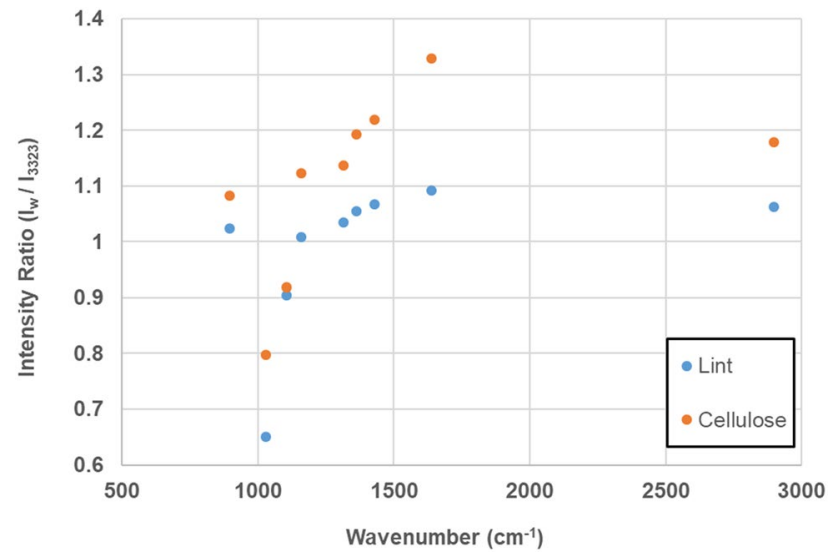

Fig. 5 Comparison of ATR-FTIR Intensity ratios of lint and cellulose fibers from IRUG database $\left(I_{w}\right.$ and $I_{3323}$ refer to the peak intensity values at any wave number and at $3323 \mathrm{~cm}^{-1}$, respectively)

the structure of the $\mathrm{C}-\mathrm{OH}$ bond present in the cellulose content to break.[1].

On comparing ATR-FTIR spectrum of lint and polyester standard fibers, it was inferred that peak intensity ratio between $I_{1711}$ and $I_{718}$ remained constant, thereby proving that the residual content in lint was in fact polyester fabric fibers. A significant reduction in ester groups resulted in low peak intensities at wavenumbers 1711 and $718 \mathrm{~cm}^{-1}$.

\subsection{XRD analysis}

Most of the textile fibers are a mixture of crystalline and amorphous parts and hence X-rays diffracted or reflected from the material will yield patterns related to the crystalline and amorphous areas within a fiber. Figure 6 shows a plot of diffraction intensity collected from the lint sample against diffraction angle $2 \theta$. The diffractogram of the lint sample depicts a typical crystalline structure character and clearly shows five distinguishable peaks at diffraction angles of $14.7^{\circ}, 16.6^{\circ}, 20.6^{\circ}, 22.5^{\circ}$, and $34.7^{\circ}$ corresponding to five Bragg angle characteristic lattice planes, namely (101), (101), (021), (002), and (040) of cellulose fibers. These diffraction peaks are taken as basis to resolve the spectrum and compare with virgin fibers reproduced from [7] shown as an inset in Fig. 6. XRD spectrum of lint is exactly similar to that of cellulose I polymorph with the peak resulting from the (002) lattice plane reflection seems to be the most intense interference of all diffraction spectral peaks.

The crystallinity index of the lint sample was evaluated using the XRD peak height method described earlier. Although in the diffractogram $\mathrm{I}_{\mathrm{AM}}$ (corresponding to $2 \theta$ of $18^{\circ}$ ) is not aligned horizontally with $I_{002}$, the peak height method provides only an approximate estimate of the contribution of crystalline cellulose to the cellulose diffraction spectrum. Also, the contribution of amorphous cellulose at $2 \theta$ of $18^{\circ}$ was assumed to be negligible in comparison to the (002) lattice plane peak. Based on Eq. (1), the crystallinity index was calculated to be around $76 \%$ which is in range with the crystallinity index values of raw cotton (73-80\%) [18] and can reach as high as $91 \%$ for bleached cotton [17]. The corresponding $d$-spacing and the crystallite size perpendicular to (002) plane are $3.9 \AA$ and $11.5 \mathrm{~nm}$, respectively which are significantly different than cellulose / polymorph values of $6 \AA$ and $5.4 \mathrm{~nm}$. This indicates that the loss in crystallinity has been due to the degradation of smaller crystallites and the growth of the defective crystallites [19] while the large crystallites have been unaffected. Moreover, the large crystallite size of lint fibers in comparison to virgin fibers could be attributed to the repeated horrification of fibers [14].

Despite chemical treatment during laundering, the lint fibers appear to contain significant proportions of crystalline region as evidenced by the value of crystallinity index. The repeated wetting and drying processes do not seem to affect the crystalline structure of the cellulosic fibers significantly. Moreover, as shown in the XRD diffractogram of
Fig. 6 X-ray diffraction spectra of lint (red line) compared with cellulose I polymorph (black line) taken from Keshk et al. [7]

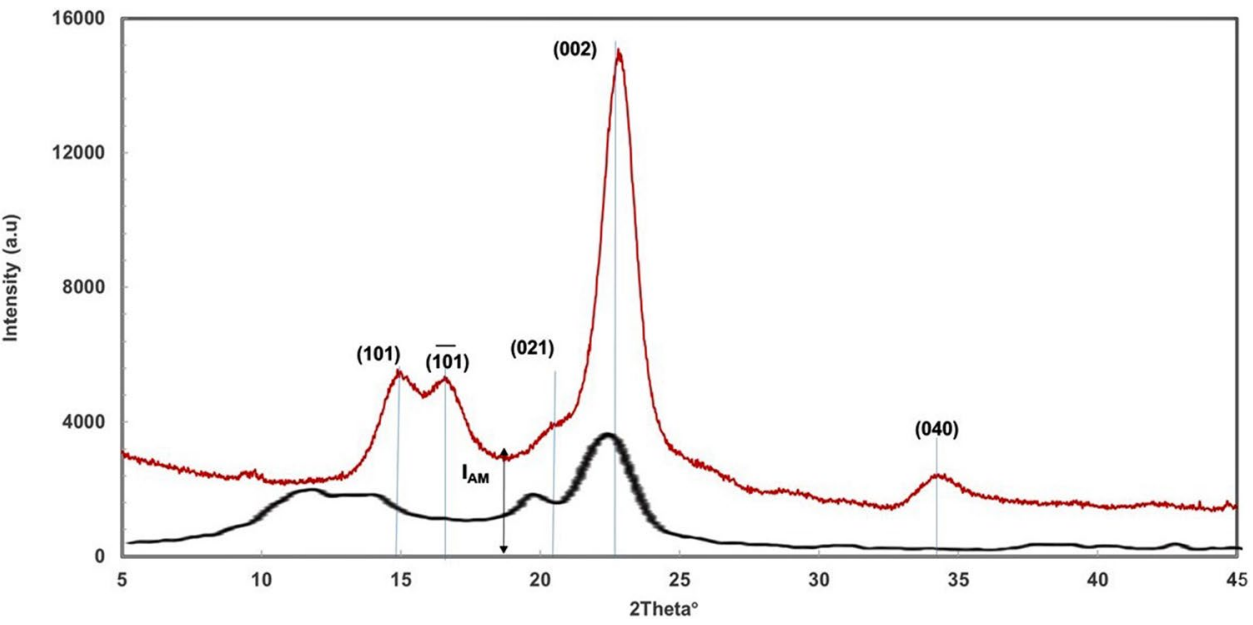

SN Applied Sciences 
the lint sample, no other impurity peaks related to laundry detergents or other contaminants are present.

Detailed ultrastructural analysis of dry lint fibers revealed the presence of microfibrils that are possibly formed by the agglomeration of elementary fibrils. The links between the crystalline and noncrystalline regions of the microfibrils are severely affected by the repeated laundering process. This is evidenced by the reduced water retention value of the bulk lint fibers as the collapse of cell walls prohibited water absorbency. It can be concluded that the laundering processes affected bulk characteristics of the lint fibers, including aspect ratio and WRV that would potentially impact sorbent properties. Results presented in this work serves to recognize lint as a valuable cellulosic feedstock for secondary recycling applications.

\section{Conclusion}

Fabric lint discarded from commercial laundry dryer filters is composed of various blends of natural and synthetic fibers. Due to the chemical processes encountered in wet laundering, the inherent properties of fibers are severely affected. In this preliminary study, the residual physical and structural characteristics of dryer lint was evaluated. Results from water retention value test and microscopic studies reveal that lint fibers are extremely short, and fibrils are agglomerated. Interestingly, spectroscopic and diffraction studies confirm that the lint fibers are semicrystalline and largely exhibit cellulose / polymorph molecular structure. The residual characteristics evaluation from this study encourages the use of resource recovery strategies for laundry lint fibers.

Although the volume of dryer lint waste generated is not significant enough for use in large-scale applications, such as solid-fuel or waste-to-energy production, it could still be engineered to manufacture high-value-added products, like adsorbents and membranes for environmental remediation.

Acknowledgements The authors would like to express sincere appreciation to Nahla Medical Supplies, Abu Dhabi for extending their support in flow microscopy analysis.

Author contributions Pravin Kannan: Methodology, Investigation, Writing-Original draft, Reviewing \& Editing, Formal analysis, validation. Fawzi Banat: Conceptualization, Formal analysis, WritingReviewing \& Editing, Resources, Supervision, Project administration.

\section{Compliance with ethical standards}

Conflict of interest The authors declare that they have no conflict of interest.
Ethical approval The authors declare that the samples used in the study were collected with appropriate permissions from supervisors/ managers of commercial laundromats and have been used solely for research purposes.

\section{References}

1. Bakri MK, Jayamani E (2016) Comparative Study of Functional Groups in Natural Fibers: Fourier transform Infrared Analysis (FTIR). In: Chauhan PS (ed) Futuristic Trends in Engineering, Science, Humanities, and Technology, 1st edn. TRO India, pp 167-174

2. Boucher J, Friot D (2017) Primary microplastics in the oceans: a global evaluation of sources. IUCN, Gland. https://doi. org/10.2305/IUCN.CH.2017.01.en

3. Cesa FS, Turra A, Baruque-Ramos J (2017) Synthetic fibers as microplastics in the marine environment: a review from textile perspective with a focus on domestic washings. Sci Total Environ 598:1116-1129

4. Das K, Ray D, Bandyopadhyay NR, Sengupta S (2010) Study of the properties of microcrystalline cellulose particles from different renewable resources by XRD, FTIR, nanoindentation, TGA and SEM. J Polym Environ 18:355-363

5. Gago J, Carretero O, Filgueiras AV, Viñas L (2018) Synthetic microfibers in the marine environment: a review on their occurrence in seawater and sediments. Mar Pollut Bull 127:365-376

6. Henry B, Laitala K, Klepp IG (2019) Microfibres from apparel and home textiles: prospects for including microplastics in environmental sustainability assessment. Sci Total Environ 652:483-494

7. Keshk SMAS, Yahia IS (2018) Physicochemical characterization of different cellulose polymorphs/graphene oxide composites and their antibacterial activity. Turk J Chem 42(2):562-571

8. Ko YC, Park JM (2015) Engineering cellulose fibers for highvalue added productsffor pulp \& paper industry. J Korea TAPPI 47(6):22-40

9. Ladewig SM, Bao S, Chow AT (2015) Natural fibers: a missing link to chemical pollution dispersion in aquatic environments. Environ Sci Technol 49(21):12609-12610

10. Lawrence CA (2010) Advances in yarn spinning technology. Woodhead Publishing, Cambridge

11. Lv X, Dong Q, Zuo Z, Liu Y, Huang X, Wu WM (2019) Microplastics in a municipal wastewater treatment plant: fate, dynamic distribution, removal efficiencies, and control strategies. J Clean Prod 225(18):579-586

12. Maiti S, Jayaramudu J, Das K, Reddy SM, Sadiku R, Ray SS, Liu D (2013) Preparation and characterization of nano-cellulose with new shape from different precursor. Carbohydar Polym 98(1):562-567

13. Mandal A, Chakrabarty D (2011) Isolation of nanocellulose from waste sugarcane bagasse (SCB) and its characterization. Carbohyd Polym 86(3):1291-1299

14. Newman RH (2004) Carbon-13 NMR evidence for cocrystallization of cellulose as a mechanism for hornification of bleached kraft pulp. Cellulose 11(1):45-52

15. Okubayashi S, Bechtold T (2005) A pilling mechanism of man-made cellulosic fabrics-effects of fibrillation. J Text Res 75(4):288-292

16. Palme A, Idström A, Nordstierna L, Brelid H (2014) Chemical and ultrastructural changes in cotton cellulose induced by laundering and textile use. Cellulose 21:4681-4691 
17. Parikh DV, Thibodeaux DP, Condon B (2007) X-ray crystallinity of bleached and crosslinked cottons. J Text Res 77(8):612-616

18. Sarna E, Włochowicz A (2003) Changes in the superstructure and surface morphology of cotton during spinning. J Text Res 73(3):216-220

19. Tang LG, Hon David NS, Pan SH, Zhu YQ, Wang ZW, Zhen Z (1996) Evaluation of microcrystalline cellulose. I. Changes in ultrastructural characteristics during preliminary acid hydrolysis. J Appl Polym Sci 59:483-488

20. Vahur S, Teearu A, Peets P, Joosu L, Leito I (2016) ATR-FT-IR spectral collection of conservation materials in the extended region of $4000-80 \mathrm{~cm}^{-1}$. Anal Bioanal Chem 408:3373-3379

21. Van Amber RR, Niven BE, Wilson CA (2010) Effects of laundering and water temperature on the properties of silk and silk-blend knitted fabrics. J Text Res 80:1557-1568
22. Wu WM, Yang J, Criddle CS (2016) Microplastics pollution and reduction strategies. Front Environ Sci Eng 11(1):6-7

23. Zambrano MC, Pawlak JJ, Daystar J, Ankeny M, Cheng JJ, Venditti RA (2019) Microfibers generated from the laundering of cotton, rayon and polyester based fabrics and their aquatic biodegradation. Mar Pollut Bull 142:394-407

24. Zhang Z, Chen Y (2020) Effects of microplastics on wastewater and sewage sludge treatment and their removal: a review. Chem Eng J 382:122955

25. Zhang W, Okubayashi S, Bechtold T (2003) Modification of fibrillation by textile chemical processing. Lenzing Ber 82:58-63

Publisher's Note Springer Nature remains neutral with regard to jurisdictional claims in published maps and institutional affiliations. 